\title{
Doctor of Dental Surgery
}

National Cancer Institute

\section{Source}

National Cancer Institute. Doctor of Dental Surgery. NCI Thesaurus. Code C39382.

The Doctor of Dental Surgery degree is the first-professional, postgraduate degree awarded for graduates who have completed a program of studies in dental surgery. 